Artigo

\title{
Estudo da Variabilidade Temporal da Profundidade Óptica do Aerossol Utilizando Dados de Sensoriamento Remoto Sobre a Região de Transição entre a Floresta Amazônica e o Cerrado
}

\author{
Nathália Velloso Prado ${ }^{1}$, Simone Marilene Sievert da Costa Coelho ${ }^{1,2}$ \\ ${ }^{1}$ Instituto Nacional de Pesquisas Espaciais, Cachoeira Paulista, SP, Brasil. \\ ${ }^{2}$ Centro de Previsão de Tempo e Estudos Climáticos, Cachoeira Paulista, SP, Brasil.
}

Recebido em 28 de Junho de 2016 - Aceito em 14 de Março de 2017.

\begin{abstract}
Resumo
O objetivo deste trabalho é apresentar uma análise observacional da variabilidade temporal da profundidade óptica do aerossol, explorando produtos de sensoriamento remoto. Neste sentido, analisou-se como déficits de chuva na estação seca impactam as atividades de queimadas na Amazônia Legal e como estas podem afetar a composição da atmosfera. Foram utilizados dados de profundidade óptica do aerossol (AOD) do sensor MODIS/Terra e da AERONET, precipitação do satélite TRMM, e, para queimadas, dados produzidos pelo CPTEC/INPE, durante 2000 a 2012. Resultados indicaram que os valores de AOD inferidos por satélite e superfície apresentaram uma tendência negativa nos últimos cinco anos da série histórica analisada, possivelmente associada às mudanças nas atividades antrópicas na região. Para este mesmo período, as taxas de desmatamento na Amazônia Legal estimadas por satélite também apresentaram redução, podendo justificar parte das mudanças na AOD. Dados de satélite indicam que o déficit de chuva na estação seca é uma das possíveis causas do aumento de queimadas em Setembro, porém não é fator único. A correlação encontrada entre dados de precipitação acumulada e focos de queimadas é inferior a -0.3. Por outro lado, a correlação entre precipitação e aerossóis é mais evidente $(R=0.7)$, explicando apenas parte da variabilidade da AOD.
\end{abstract}

Palavras-chave: queima de biomassa, aerossóis atmosféricos, precipitação, taxa de desmatamento.

\section{Study of Aerosol Optical Depth Temporal Variability Using Remote Sensing Data in a Transition Region Between Amazon Forest and Brazilian's Savannah}

\begin{abstract}
This work aims to present an observational analysis about the temporal variability of aerosol optical depth (AOD), exploring remote sensing products. It is studied how rain deficits in dry season can impact fire activities in Legal Amazon, and how they could affect the atmosphere's composition. It is used AOD data from MODIS/Terra sensor and AERONET network station, for precipitation we use TRMM satellite data, and for biomass burning, data products from CPTEC/INPE ranging from 2000 to 2012. Results indicate that AOD values inferred from satellite and AERONET present a negative trend in the last five years, probably associated with changes in the anthropic activities in the region. For the same period, the deforestation rate in Legal Amazon estimated from satellite also present reduction. Satellite data indicate that rain deficit during dry season is one of the possible causes for burn increasing in September, but is not the only factor. The correlation between accumulated precipitation data and burns founded in this study is lower than -0.3 . By the other hand, the correlation between precipitation and aerosols is more pronounced $(R=0.7)$, but explains just part of the AOD variability. Deforestation decreasing on such region could also justify the AOD changes.
\end{abstract}

Keywords: biomass burning, atmospheric aerosols, precipitation, deforestation rate.

Autor de correspondência: Nathália Velloso Prado, nathalia.velloso@cptec.inpe.br. 


\section{Introdução}

Os aerossóis são partículas sólidas ou líquidas suspensas na atmosfera oriundas de diversas fontes naturais e antropogênicas, tais como poeira mineral do solo, sal marinho, fuligem e gases de erupções vulcânicas, material biogênico de florestas, dentre outras (Correia et al., 2013). No Brasil, a ação antrópica de queima de biomassa é muito utilizada como forma de cultura de preparação do solo para a prática agropecuária, sendo uma das principais fontes de aerossóis.

Dependendo da concentração e composição química, os aerossóis podem afetar a qualidade do ar, o balanço radiativo, as características microfísicas das nuvens e a eficiência (supressão e/ou intensificação) da precipitação (Artaxo et al., 1998; Rosenfeld, 1999).

Considerando a interação não linear entre aerossol nuvem - precipitação, quantificar os efeitos diretos e indiretos do aerossol no clima regional e global vem sendo um dos grandes desafios para a comunidade científica (Intergovenamental Panel of Climate Change (IPCC), 2013). Avanços no monitoramento contínuo de aerossóis através de medições à superfície e de sensoriamento remoto, integrados à modelagem ambiental, vêm permitindo uma melhor compreensão destas incertezas quanto a estes efeitos. No Brasil, existem duas importantes bases de dados históricos de aerossóis, a da rede da AERONET - AErosol RObotic NETwork, (Holben et al., 1998) e a dos produtos do sensor MODIS (MODerate Resolution Imaging Spectroradiometer) a bordo dos satélites Terra e Aqua (Anderson et al., 2003), cujos produtos de aerossóis cobrem diariamente todo o globo e são disponibilizados pela NASA (National Aeronautics and Space Administration). A AERONET, uma rede global concebida pela NASA/GSFC (Goddard Space Flight Center), é mantida por institutos de ensino e pesquisa e possui instrumentos padronizados, chamados fotômetros solares. Estes medem a radiância solar direta incidente à superfície e a radiância solar difusa proveniente de distintas direções celestes e em diversos comprimentos de onda no intervalo espectral de 0.4 a $1.6 \mu \mathrm{m}$, o que permite inferir propriedades físicas de aerossóis, tais como distribuição de tamanho, profundidade óptica, índice de refração complexo, entre outros. O Brasil e os Estados Unidos foram os primeiros a compor a rede em 1993, cada país com oito estações. Atualmente, a rede possui mais de 250 estações distribuídas pelo globo e são usadas como referência terrestre na caracterização do aerossol.

Considerando a importância dos aerossóis de queimadas no clima regional, diversos estudos no país avaliaram o impacto destes na precipitação (Camponogara; Silva Dias; Carrió, 2014; Coelho; Costa; Freitas, 2013; Coelho et al., 2012; Torres et al., 2010; Vendrasco; Silva Dias; Freitas, 2009), na radiação (Procopio, S. et al., 2004; Rosário et al., 2011; Sena; Artaxo; Correia, 2013; Yamasoe et al., 2006), na saúde humana (Goto et al., 2011; Pires-Neto; Saldiva;
Dolhnikoff, 2007; Silva et al., 2012; Sisenando et al., 2012, 2011) e na qualidade do ar (Freitas et al., 2005, 2011; Longo et al., 2013; Pereira et al., 2011).

A concentração e composição química do aerossol presente na atmosfera depende de diversos fatores, entre eles, fatores meteorológicos associados a circulação, estabilidade, remoção e umidade (Rosário et al., 2011). As principais regiões emissoras de aerossóis de queimadas têm se concentrado na Amazônia Legal, apresentando uma forte e permanente dinâmica no uso e mudança do solo (Escada; Alves, 2001). Estudos envolvendo a mencionada região, (Alves et al., 1998; Escada; Alves, 2001; Instituto Nacional de Pesquisas Espaciais (INPE), 1989, 1999, 2000; PRODES, 1996) apontam que algumas áreas dos estados do Acre, Rondônia, Maranhão, Mato Grosso e Pará apresentam as taxas mais altas de desflorestamento e de mudanças constantes no uso do solo. Apesar disso, essas regiões demonstram características distintas em seus históricos de evolução de ocupação e uso da terra (Becker, 1997, 1998; Godfrey; Browder, 1996).

No caso do Mato Grosso, algumas das suas terras nativas foram desmatadas por meio da derrubada da floresta. Inicialmente, para implantação da pecuária e, em seguida, transformadas em áreas de agricultura mecanizada, principalmente para o cultivo de soja, fazendo uso de fertilizantes e de sementes modificadas geneticamente (Domingues; Bermann, 2012).

Historicamente, o processo de evolução da produção de soja no país ocorreu na segunda metade de 1970 e, a partir da década de 80 , houve a expansão para áreas de cerrado. Neste período, diversos incentivos do governo brasileiro (EMBRAPA, 2013) foram feitos até que, em 1990, o estado do Mato Grosso se tornou o terceiro maior produtor de soja do Brasil. As diversas fases de ocupação do solo e desenvolvimento socioeconômico no Mato Grosso poderiam, juntamente com fatores climáticos, contribuir para a variabilidade temporal dos cenários de emissões de aerossóis.

Alguns estudos, como o de Koren, Remer e Longo (2007) observaram uma tendência de aumento nos focos de queimadas obtidos via sensoriamento remoto na Amazônia para o período de 1998 a 2005, tendo uma inversão abrupta no ano seguinte, 2006. Além disso, outros trabalhos com relação às análises de tendências de propriedades ópticas do aerossol em outras regiões também já foram discutidos na literatura (Levy et al., 2015; Li et al., 2014; Lyapustin et al., 2011; Zhang; Reid, 2010). Em um trabalho recente, Li et al. (2014) apresentaram uma avaliação de tendências de AOD e de outros parâmetros ópticos inferidos via AERONET tendo a América do Norte, Europa e Japão como principais áreas de estudo. No Brasil, os referidos autores mostraram que existe uma tendência negativa na AOD em $440 \mathrm{~nm}$ para as estações de Cuiabá - Miranda e Alta Floresta. Outro importante trabalho foi conduzido por Zhang et al. (2010), o qual teve como foco a tendência de 
aerossóis sobre regiões oceânicas. Estes trabalhos apenas indicaram a existência da tendência negativa, sem relacionar com fatores antrópicos e climáticos.

O presente estudo tem por objetivos efetuar uma análise observacional da variabilidade temporal de AOD para o período de 2000 a 2012 e verificar a possível relação entre déficit de chuva na estação seca, número de focos de queima e carga de aerossóis. Distinto de outros trabalhos, que geralmente avaliam o impacto dos aerossóis na precipitação da estação chuvosa, este pretende avaliar a relação precipitação, atividades antrópicas e aerossóis durante o período de estação seca que antecede o pico das queimadas. Para tanto, o trabalho explora o uso de dados de sensoriamento remoto inferidos via superfície e satélite, provenientes de distintos sensores. A área de estudo escolhida situa-se em uma região de transição entre a floresta amazônica e o cerrado. Compreende ainda praticamente todo o estado do Mato Grosso, o qual faz parte da Amazônia Legal e compõe o chamado Arco do Desflorestamento.

\section{Metodologia}

A motivação do presente estudo observacional foi explorar o potencial das informações de sensores a bordo de satélite na avaliação do impacto da precipitação e do desmatamento nas atividades de queimadas e, consequentemente, na poluição do ar. Particularmente, procurou-se verificar, por meio de dados de sensoriamento remoto de distintos sensores satelitais, a possível relação entre déficit de chuva na estação seca, número de focos de queima e a carga de aerossóis.

Os dados mensais de focos de queimadas utilizados são produzidos pelo INPE e são inferidos a partir de informações dos sensores AVHRR (Advanced Very High Resolution Radiometer) e MODIS, respectivamente, a bordo dos satélites da série NOAA e Aqua utilizando técnicas de limiares para os canais do infravermelho solar e termal (3.9 e $10 \mu \mathrm{m})$ (Setzer, 2007). A resolução espacial do produto de focos queimadas é de $1 \mathrm{~km}$.

A concentração de aerossol é inferida por meio dos dados mensais de profundidade óptica em $550 \mathrm{~nm}$ (AOD, em inglês Aerossol Optical Depth) do sensor MODIS/Terra, cuja resolução espacial é de $100 \mathrm{~km}$, é proveniente da base de dados da NASA, GIOVANNI. Adicionalmente, dados de AOD em $500 \mathrm{~nm}$ da AERONET também são utilizados para o sítio de Alta Floresta. A profundidade óptica do aerossol é um parâmetro físico adimensional e indica o quanto o feixe de radiação é atenuado pelos aerossóis à medida que se propaga em uma determinada camada da atmosfera que contenha aerossóis.

A taxa de desmatamento é proveniente do banco de dados do PRODES (Projeto de Monitoramento do Desmatamento na Amazônia Legal por Satélite), disponível pelo INPE, o qual já está estabelecido na literatura (Kintisch, 2007). Estes dados são estimados a partir dos incrementos anuais de desmatamento de áreas em que há ocorrência de remoção completa da cobertura florestal. A estimativa de desmatamento é através de imagens dos satélites Landsat, complementadas com imagens do DMC-UK (Disaster Monitoring Constellation - United Kingdom) e CBERS (China-Brazil Earth-Resources Satellite), com resolução espacial de 20 a $30 \mathrm{~m}$ e taxa de revisita de 16 dias, garantindo assim, minimizar o problema da cobertura de nuvens (Instituto Nacional de Pesquisas Espaciais (INPE), 2013).

Para precipitação, são utilizados os produtos 3B43 do satélite TRMM, Tropical Rainfall Measuring Mission, (Kummerow et al., 2000) disponibilizados pelo sistema de distribuição da NASA (DAAC, Distributed Active Archive System). O produto de precipitação tem resolução de aproximadamente 0,25 graus (aproximadamente $25 \mathrm{~km}$ no Equador) e combina diferentes sensores a bordo do TRMM (TMI - Microwave Imager, PR - Precipitation Radar e VIRS - Visible and Infrared Scanner) e dados de estações pluviométricas na superfície (Huffman et al., 2007).

Dados de área plantada de soja no estado do Mato Grosso provenientes do levantamento oficial do IBGE (Instituto Brasileiro de Geografia e Estatística) também são utilizados para descrever as atividades agrícolas da região.

Inicialmente, a variabilidade sazonal e interanual dos aerossóis via sensor MODIS e da precipitação é analisada sobre uma área contida em um quadrado de $\left[10^{\circ}\right.$ a $\left.17^{\circ} \mathrm{S}\right]$ de latitude e $\left[50^{\circ}\right.$ a $\left.60^{\circ} \mathrm{W}\right]$ de longitude, ocupando praticamente todo o estado do MT e áreas vizinhas (Fig. 1). Dados de focos de queimadas são analisados para a região da Amazônia Legal e o MT, separadamente. A sazonalidade das atividades de queimadas, a presença de aerossol na atmosfera e a precipitação são estudadas durante o período de 2000 a 2012. A escolha do período está associada à disponibilidade do conjunto de dados de sensoriamento remoto. A base de dados do produto de AOD MODIS/Terra tem início em Fevereiro de 2000, enquanto que os dados de precipitação do TRMM tem início em Janeiro de 2000. Para ambos, a análise é efetuada até Dezembro de 2012.

Em seguida, a relação entre a precipitação, queimadas e a profundidade óptica do aerossol é estudada. A escolha desta região justifica-se por apresentar uma das principais fontes de aerossóis no Brasil, provenientes de atividades antrópicas. Análises estatísticas padrões, como coeficiente de correlação e regressão linear são aplicadas para determinar a possível relação entre precipitação, $\mathrm{AOD}$ e queimadas. Adicionalmente, para relacionar as diversas variáveis, é estimada a anomalia padronizada através da diferença entre média mensal e sua média climatológica (2000-2012) dividida pelo desvio padrão. As estatísticas são calculadas usando as médias de AOD/MODIS e precipitação numa região abrangendo $12.5 \times 10^{3} \mathrm{~km}^{2}$, e, para as queimadas, os dados copilados pelo INPE para o estado do MT.

Por fim, é realizada uma comparação entre os dados de AOD observados para a área da região estudada via sen- 
sor orbital MODIS e via superfície (AERONET), para o sítio de Alta Floresta $\left(09^{\circ} 55^{\prime} \mathrm{S}, 56^{\circ} 00^{\prime} \mathrm{W}\right)$, localizada na porção norte do estado do Mato Grosso, região fronteiriça com o Arco do Desflorestamento (Fig. 1). Os dados provenientes da AERONET são selecionados baseados no mesmo horário de passagem do satélite Terra nessa localidade, aproximadamente 13:30 UTC. A escolha desse horário permite verificar se estas duas bases de dados de AOD reproduzem resultados semelhantes em termos de tendência.

\section{Resultados e Discussões}

3.1. Variabilidade sazonal e interanual de queimadas, aerossóis e precipitação

A Fig. 2 mostra a variabilidade interanual de AOD, da precipitação e do número acumulado de focos de queimadas no período de Janeiro de 2000 a Dezembro de 2012. A região possui um ciclo anual de chuva marcante com regime dividido por estação seca e úmida. Observações de satélite indicam precipitação média mensal superior a $200 \mathrm{~mm}$ no período de Novembro a Março, e inferior a 50 $\mathrm{mm}$ nos meses subsequentes até início da transição da estação chuvosa em Outubro (Tabela 1). Este regime obtido via satélite está de acordo com os resultados da literatura que utilizam dados observacionais de estações meteorológicas (Reboita et al., 2010).

Durante a estação seca, ocorre um aumento expressivo das atividades de queima (Fig. 2c). O número de queimadas média mensal na Amazônia Legal no período de 2000 a 2012 (Tabela 1) é da ordem de $10^{3}$ no primeiro trimestre e sobe para valores superiores a $10^{5} \mathrm{em}$ Agosto e

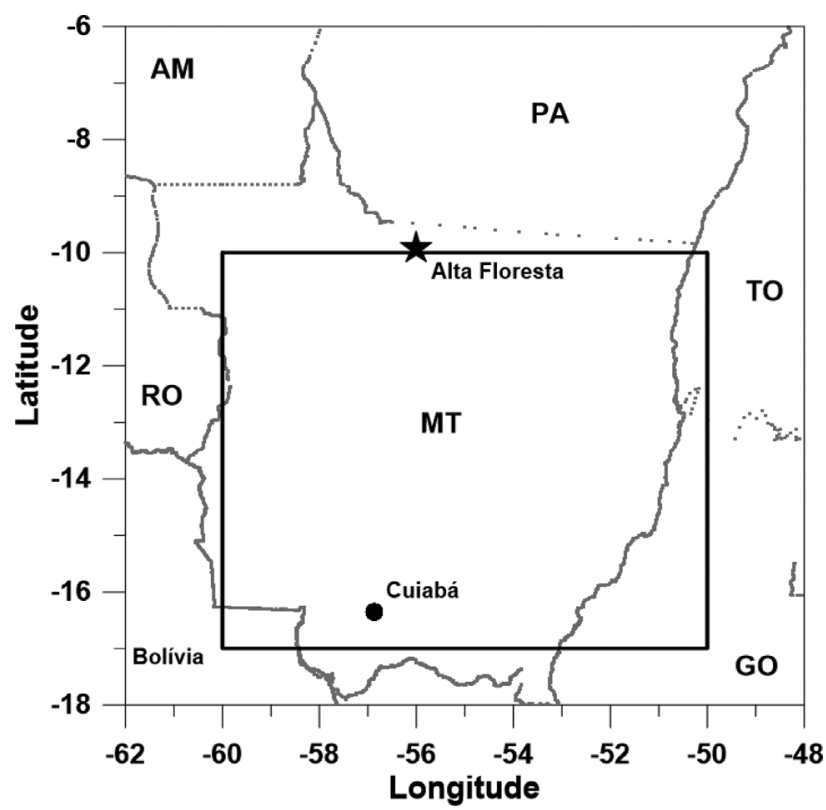

Figura 1 - Região de estudo centrada no quadrado cobrindo $\left[10^{\circ}\right.$ a $\left.17^{\circ} \mathrm{S}\right]$ de latitude e $\left[50^{\circ}\right.$ a $\left.60^{\circ} \mathrm{W}\right]$ de longitude e a localização do sítio de Alta Floresta $\left(09^{\circ} 55^{\prime} \mathrm{S}, 56^{\circ} 00^{\prime} \mathrm{W}\right)$ da AERONET.
Setembro. No início da série temporal, o número de queimadas era inferior a $50 \times 10^{3}$, aumentando gradativamente nos anos subsequentes, atingindo valores de aproxima-

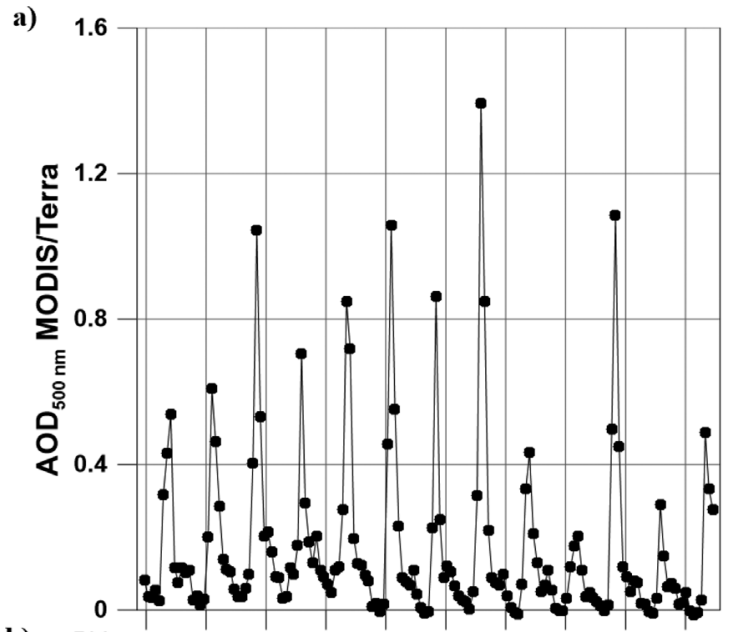

b)
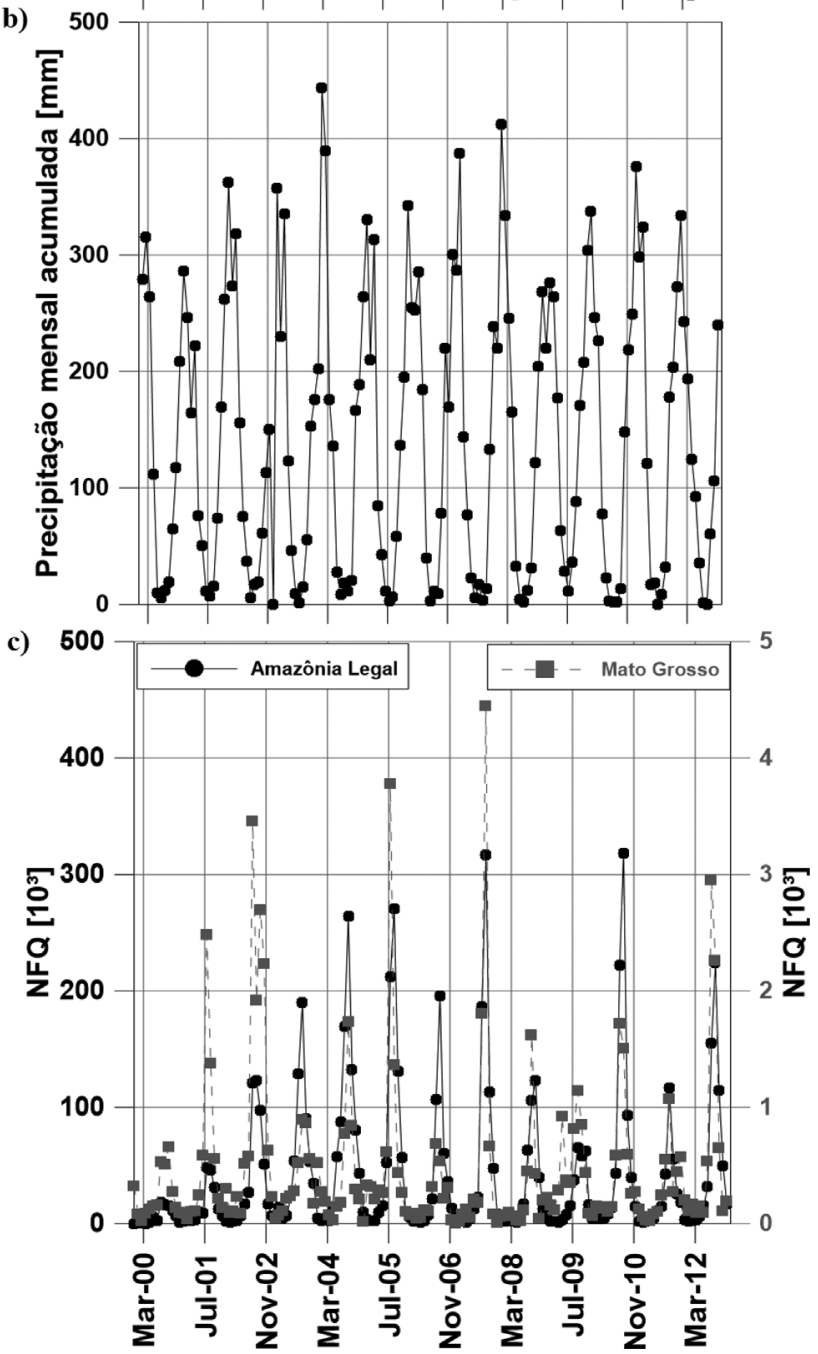

Figura 2 - Série temporal (2000 - 2012) de: a) $\mathrm{AOD}_{550 \mathrm{~nm}}$ mensal via sensor MODIS/Terra; b) Precipitação mensal acumulada em milímetros e c) Número de Focos de Queima (NFQ) para a Amazônia Legal e o estado do Mato Grosso (103). 
Tabela 1 - Médias climatológicas (2000-2012) de precipitação e AOD para a região de estudo e número de focos de queima na Amazônia Legal obtidas por satélite.

\begin{tabular}{lccc}
\hline Meses & $\begin{array}{c}\text { Precipitação } \\
\text { TRMM (mm) }\end{array}$ & $\begin{array}{c}\text { AOD } \\
\text { MODIS/Terra } \\
\text { (adimensional) }\end{array}$ & $\begin{array}{c}\text { Número de Focos } \\
\text { de Queima } \\
\text { AVHRR/NOAA }\end{array}$ \\
\hline Jan & 312.8 & 0.10 & 3915 \\
Fev & 284.0 & 0.08 & 2733 \\
Mar & 239.0 & 0.08 & 3955 \\
Abr & 116.9 & 0.04 & 2487 \\
Mai & 35.9 & 0.02 & 5648 \\
Jun & 8.7 & 0.02 & 15714 \\
Jul & 9.4 & 0.03 & 30617 \\
Ago & 13.5 & 0.23 & 116126 \\
Set & 50.7 & 0.71 & 173272 \\
Out & 149.9 & 0.44 & 85759 \\
Nov & 201.7 & 0.18 & 43567 \\
Dez & 279.6 & 0.11 & 16797 \\
\hline
\end{tabular}

damente $300 \times 10^{3} \mathrm{em} 2007$ (seis vezes maior em relação ao início da série), diminuindo nos anos subsequentes, com exceção dos anos de 2010 e 2012 (Fig. 2c). O estado do Mato Grosso também apresenta um aumento gradativo dos focos de queimadas, com um valor máximo em Agosto de 2007 (4.5 x 103). Associado ao período de queimadas verificam-se altos valores mensais médios de AOD, (Fig. 2a). Climatologicamente, o valor de $\mathrm{AOD}_{550 \mathrm{~nm}}$ médio mensal inferido pelo satélite é inferior a $0.1 \pm 0.03$ nos primeiros seis meses do ano, apresentando eminência de aumento em Agosto, atingindo pico de $0.70 \pm 0.4 \mathrm{em}$ Setembro e diminuindo entre Outubro e Novembro, período de transição da estação seca para a chuvosa. Os anos que apresentaram os maiores valores de $\mathrm{AOD}_{550 \mathrm{~mm}} \mathrm{em}$ Setembro são $2002\left(\mathrm{AOD}_{550 \mathrm{~nm}}=1.03\right), 2005\left(\mathrm{AOD}_{550 \mathrm{~nm}}=\right.$ $1.05), 2007\left(\mathrm{AOD}_{550 \mathrm{~nm}}=1.4\right)$ e $2010\left(\mathrm{AOD}_{550 \mathrm{~nm}}=1.1\right)$. Interessante verificar que os valores de $\mathrm{AOD}_{550 \mathrm{~nm}}$ são menores nos últimos cinco anos da série temporal (com exceção de 2010), acompanhando um comportamento similar ao número de focos de queimadas. Por outro lado, tendências na série temporal de precipitação não são evidentes.

A Fig. 3 mostra a variabilidade interanual da anomalia padronizada de $\mathrm{AOD}$, do número de focos de queimadas e da precipitação acumulada. No caso da AOD é utilizado o valor máximo da média mensal dos meses de Setembro e Outubro, quando ocorre o pico da concentração dos aerossóis na atmosfera. Para as queimadas, utiliza-se a soma nos meses de Agosto a Outubro, quando ocorre o maior número de queimadas na região de estudo. Para a precipitação é usada a soma dos meses de Abril a Agosto, período que engloba desde o início da estação seca até a sua transição com a estação úmida. A chuva acumulada nestes meses é

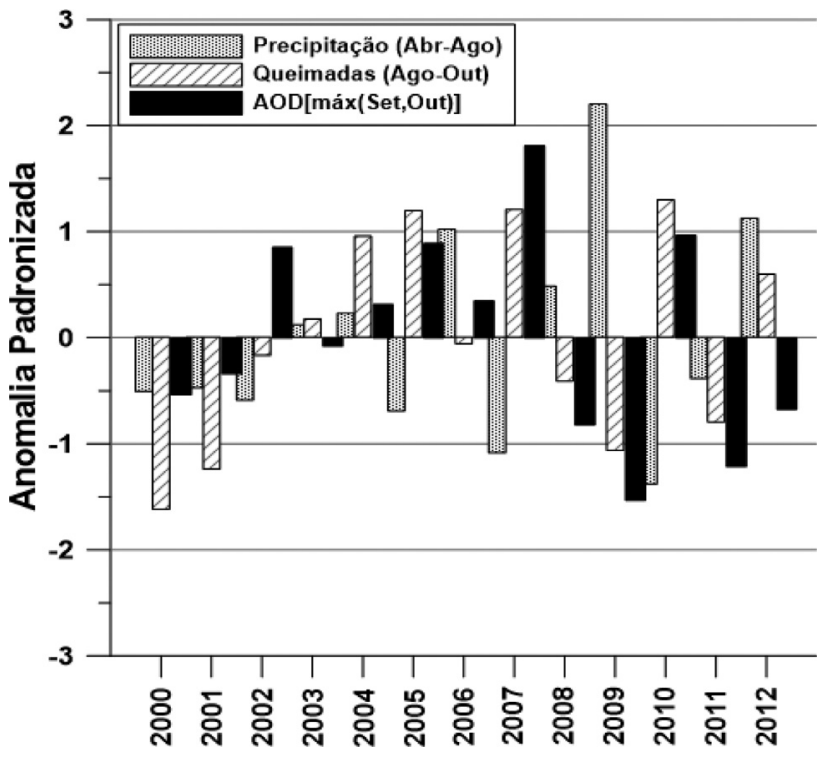

Figura 3 - Anomalia padronizada da precipitação, queimadas e AOD na região de estudo.

um dos fatores que determina a disponibilidade de água no solo e o estado da vegetação (Costa; Rodrigues, 2015), podendo impactar nas atividades de queimadas. De acordo com dados do TRMM, os anos mais secos dos últimos 13 anos apresentados neste estudo são 2005, 2007 e 2010. Esses anos apresentam anomalias negativas significativas de precipitação (i.e. anomalias acima de $1 \sigma$, em que $\sigma$ é o desvio padrão da média climatológica) em uma área de $12500 \mathrm{~km}^{2}$ durante cinco meses (Abril a Agosto), indicando seca prolongada em uma ampla área. Altos valores de AOD inferidos pelo sensor MODIS indicam que os anos de 2005, 2007 e 2010 ainda são caracterizados como os anos de maior concentração de aerossóis. Esses anos são marcados pela ocorrência do fenômeno La Niña, considerados, respectivamente, como fraco, forte e moderado (NOAA, 2015). O impacto do fenômeno La Niña acarreta diminuição das chuvas na região, e, consequentemente, reduz a umidade do solo, propiciando um ambiente com risco elevado para o fogo.

Em contraste, o ano de 2009 pode ser considerado, em relação à climatologia, o mais úmido e de menor concentração de aerossóis. A anomalia padronizada de precipitação para este ano é aproximadamente $2.3 \sigma$. Os valores de AOD inferiores a 0.2 no período de Agosto a Setembro de 2009 são similares aos valores climatológicos para o período chuvoso. Os anos de 2005, 2007, 2009 e 2010 são bons exemplos que ilustram o papel da variabilidade da precipitação na caracterização da carga de aerossóis na atmosfera.

A Fig. 3 mostra o sinal positivo da tendência dos valores de AOD no período de 2000 a 2007, invertendo o sinal nos últimos anos, com exceção do ano de 2010 . 


\subsection{Análise da relação precipitação, queimadas e aerossóis}

Na seção anterior discutiu-se a variabilidade temporal das variáveis analisadas neste estudo. Esta seção visa quantificar a relação entre AOD e precipitação acumulada, no período que antecede o pico das queimadas na região de estudo para o mês de Setembro, utilizando-se a regressão linear e a correlação, como ferramentas estatísticas. Distinto dos trabalhos apresentados na literatura que analisam o impacto dos aerossóis no regime de precipitação da estação chuvosa, este estudo faz uma avaliação observacional sobre a relação da precipitação no favorecimento das queimadas e, consequentemente, na presença de aerossóis na atmosfera.

A Fig. 4 mostra os diagramas de dispersão relacionando as anomalias de precipitação padronizada com a AOD (Fig. 4a) e a anomalia padronizada de precipitação

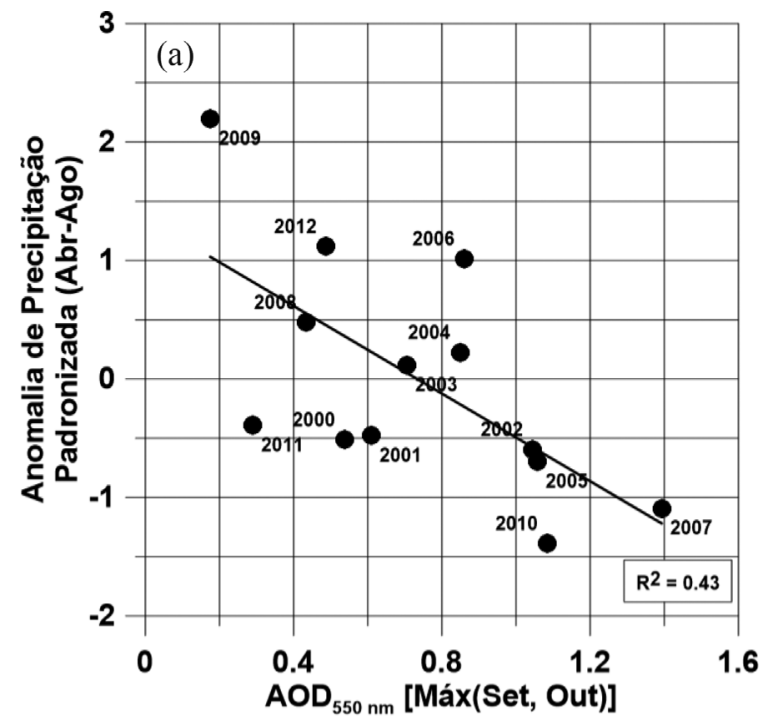

com as queimadas (Fig. 4b). Adicionalmente, a anomalia padronizada de queimadas é analisada em termos da AOD (Fig. 4c). Verifica-se que a correlação entre anomalias de precipitação e queimadas é baixa $(R=0.3)$, evidenciando que as queimadas não estão associadas apenas ao déficit de chuva, pois dependem de outros fatores não climáticos, tais como socioeconômicos.

No entanto, a profundidade óptica do aerossol aumenta monotonicamente com a diminuição da anomalia padronizada de precipitação, apresentando um coeficiente de correlação negativo de 0.7 , o que indica uma relação linear inversa entre a variabilidade do aerossol e a precipitação. Déficits de precipitação desde o início da estação seca em Abril provocam a diminuição de umidade do solo, e, consequentemente, a superfície tem maior vulnerabilidade e risco de fogo.

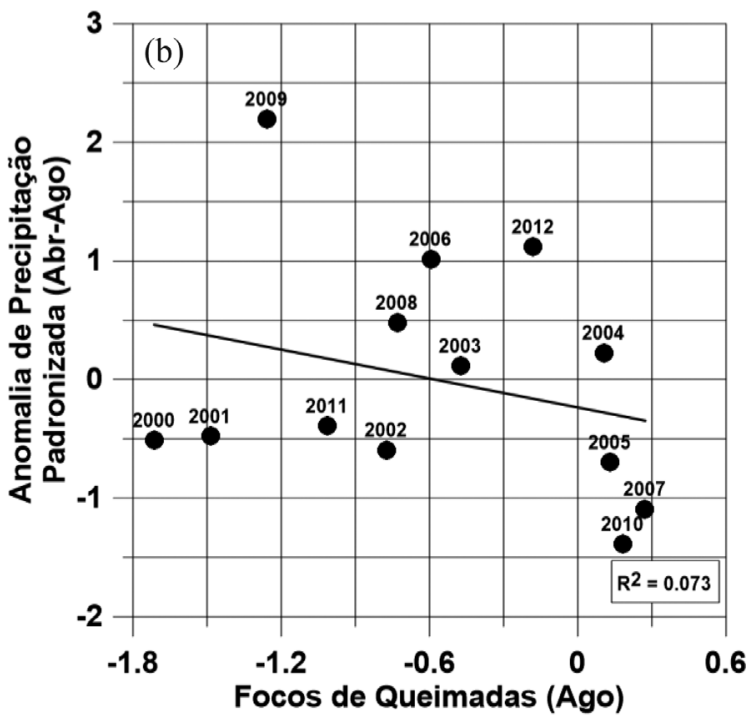

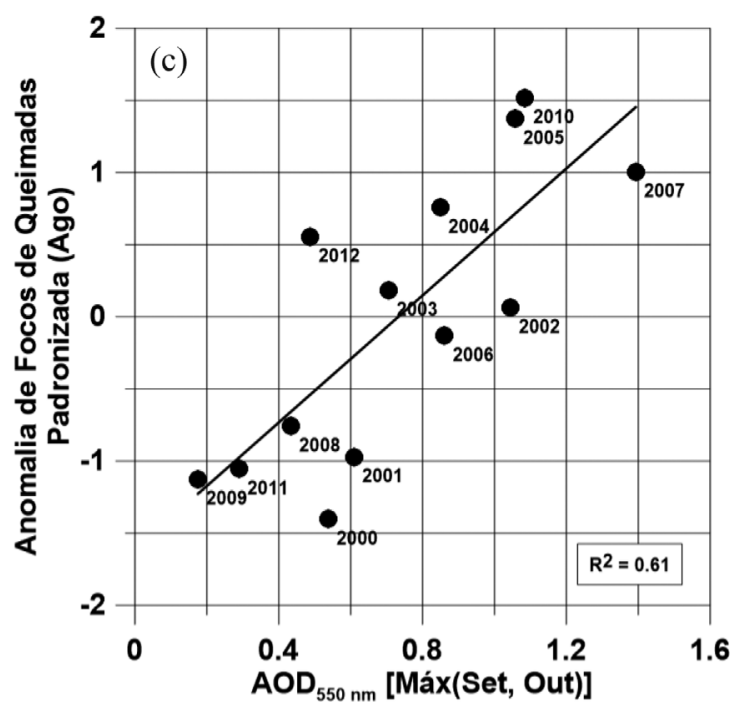

Figura 4 - Diagramas de dispersão da anomalia padronizada de precipitação acumulada no período de Abril a Agosto em relação a: (a) $\mathrm{AOD}_{550 \mathrm{~nm}} \mathrm{e}$ (b) Focos de queimadas em Agosto. (c) Diagrama de dispersão da anomalia padronizada de focos de queimadas em Agosto e AOD $_{550} \mathrm{~nm}$. 


\subsection{Análise de tendência dos dados de AOD via sensor MODIS E AERONET}

Interessante observar a tendência negativa de AOD inferida por satélite nos últimos 5 anos da série estudada sobre uma área extensa (Fig. 3). Esta tendência também é observada nos dados de $\mathrm{AOD}_{500 \mathrm{~nm}}$ do sítio da Alta Floresta na rede da AERONET (Fig. 5). Dados do sensor MODIS são incluídos nesta figura para o pixel mais próximo do

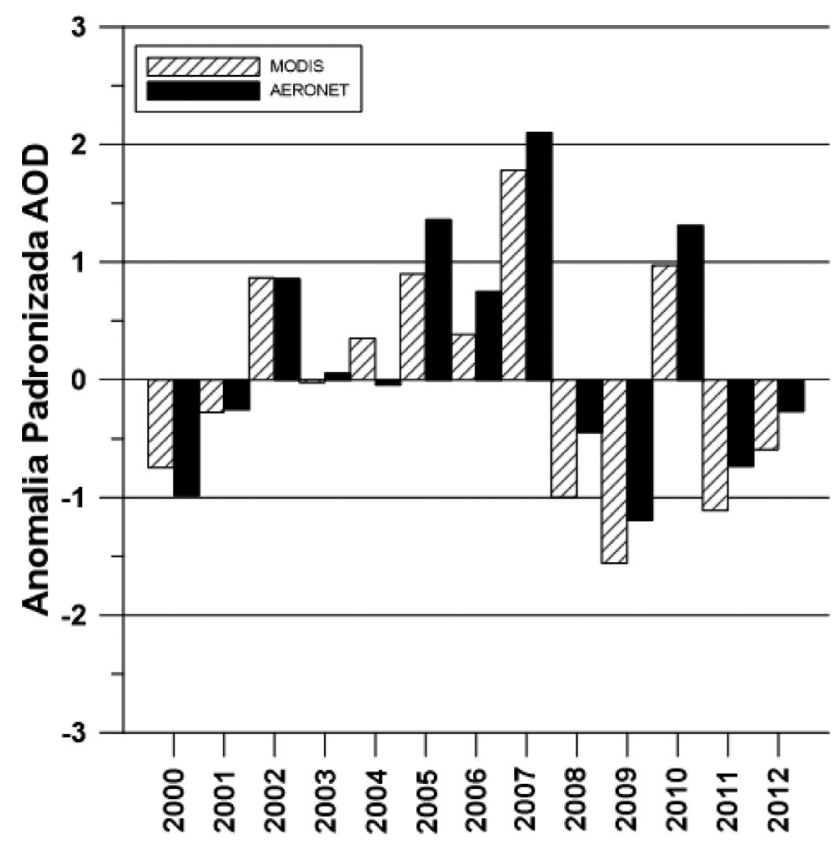

Figura 5 - Anomalia padronizada de AOD para os meses de Agosto, Setembro e Outubro via sensor MODIS/Terra para o comprimento de onda de $550 \mathrm{~nm}$ para a região compreendida no quadrado estudado e AERONET para o comprimento de onda de $500 \mathrm{~nm}$ para a estação de Alta Floresta.

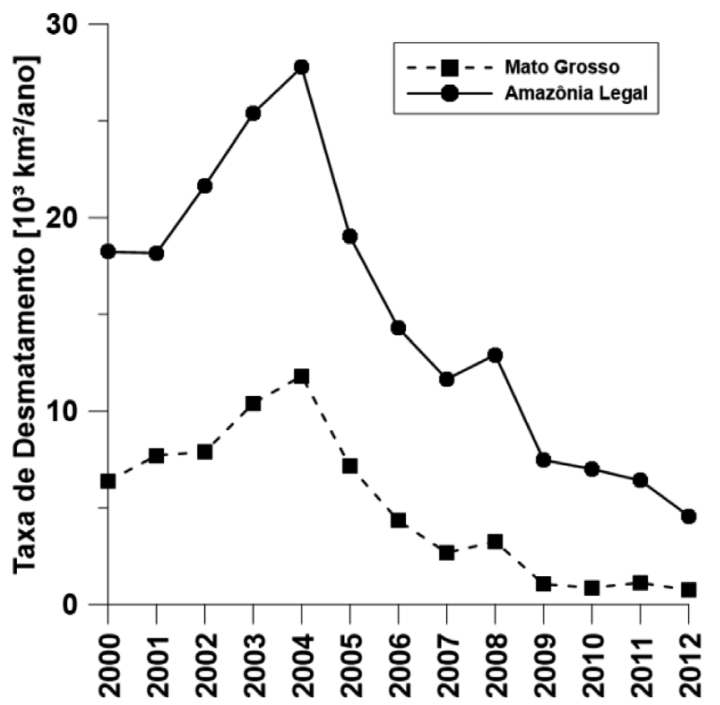

(a) sítio. Para ambas as bases de dados, observa-se uma anomalia positiva de AOD até o ano de 2007, invertendo o sinal em anos posteriores, similar ao encontrado para a grande área de estudo.

A seção anterior mostrou que a chuva pode explicar apenas parte da variabilidade do aerossol. Considerando que AOD é uma propriedade óptica, alterações em sua magnitude podem ser devido a mudanças na concentração do aerossol na atmosfera ou ainda na sua composição química e distribuição de tamanho. Mudanças no comportamento temporal de AOD possivelmente podem estar associadas às modificações na emissão do aerossol de queimadas para atmosfera, relacionada às alterações no manuseio do solo nessa região.

Essa hipótese é evidente na Fig. 6a, a qual mostra a variação temporal das taxas de desmatamento (TD) na Amazônia Legal e Mato Grosso (MT). Observa-se que a TD na Amazônia Legal tem um pico de aproximadamente $30000 \mathrm{~km}^{2} /$ ano em 2004, e diminui em anos posteriores, sendo 6 vezes menor $\left(5000 \mathrm{~km}^{2} / \mathrm{ano}\right)$ em 2012 . Por outro lado, apesar do desmatamento diminuir no estado do Mato Grosso, a área plantada vem aumentando (Fig. 6b). A redução do desmatamento na região pode explicar parte das mudanças na AOD.

Segundo Artaxo et al. (1998) as partículas de aerossóis na região Amazônica são uma combinação de diferentes processos de queima de vegetação, como cerrado, áreas de pastagem e florestas primária e secundária. Portanto, mudanças na AOD também podem ser associadas a menor contribuição do material queimado de áreas de florestas. A avaliação de possíveis mudanças nas propriedades ópticas dos aerossóis precisa ser melhor explorada utilizando outros parâmetros intrínsecos, como distribuição de tamanho e índice de refração. Outra fonte interessante para

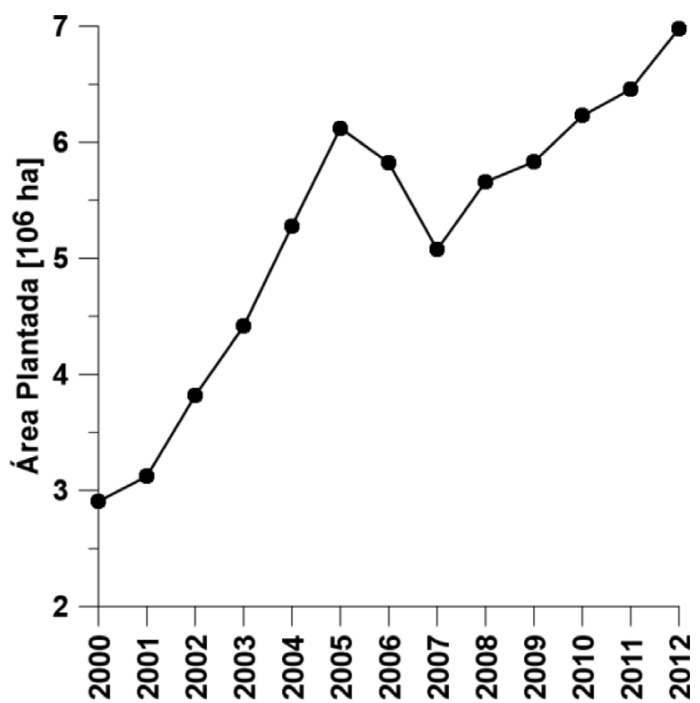

(b)

Figura 6 - (a) Taxa de desmatamento para a Amazônia Legal e o estado do Mato Grosso dado em 103 km²/ano para o período de 2000 a 2012 . Fonte: PRODES/INPE. (b) Área plantada com o cultivo de soja no estado do Mato Grosso dado em $10^{6}$ hectares para os anos de 2000 a 2012 . Fonte: IBGE. 
avaliar potenciais mudanças do aerossol na região amazônica seria a sua composição química. Portanto, dados contínuos para longos períodos são inexistentes.

\section{Discussões Finais}

Este trabalho de caráter observacional teve por finalidade explorar o potencial das informações de sensores a bordo de satélite no estudo da relação da precipitação, atividades de queimadas e poluição do ar. Particularmente, procurou-se compreender como déficits de chuva na estação seca impactam as atividades de queimadas no Mato Grosso e áreas vizinhas, e, como estas podem afetar a composição da atmosfera em termos da concentração de aerossóis.

Adicionalmente, uma possível mudança no comportamento temporal da AOD também foi estudada, explorando dados de sensoriamento remoto via satélite e via superfície. A análise entre os dados de precipitação acumulada e os dados de focos de queimadas apresentou um coeficiente de correlação inferior a -0.3 , indicando que o déficit de chuva na estação seca é uma das possíveis causas do aumento de queimadas em Setembro, porém não é fator único. As atividades socioeconômicas e políticas de preservação de florestas também apresentam um papel importante na atividade de queimada na região (Brown et al., 2006). A correlação entre o número de focos de queima e $\mathrm{AOD}_{550 \mathrm{~nm}}$ encontrada foi de 0.8 , indicando uma relação mais evidente entre queimadas e a qualidade do ar, como esperado.

Por outro lado, foi observada uma tendência de diminuição na taxa de desmatamento no ano posterior a 2004, enquanto um aumento na área plantada com o cultivo de soja neste mesmo ano foi constatado. Uma comparação entre as anomalias de AOD via sensor MODIS e AERONET apresentaram comportamento similar: uma tendência de diminuição de AOD para o período posterior a 2005. Uma das possíveis causas da diminuição na tendência de AOD poderia estar relacionada à implementação de políticas públicas de prevenção às queimadas criados em conjunto com os governos brasileiro, boliviano e peruano, como abordado por Brown et al. (2006) e Koren et al. (2007).

Entretanto, esse resultado encontrado pode causar impactos em outras propriedades ópticas do aerossol, devido a uma menor contribuição do material queimado da vegetação que compõe essa região (florestas primárias, secundárias, áreas de pastagem e cerrado).

Não obstante, estes resultados abordaram de maneira limitada a complexidade da relação entre fatores climáticos e antrópicos nas queimadas e na poluição do ar, fazendo-se necessário avançar no entendimento dessa questão. Um dos fatores climáticos importantes que não foi analisado neste estudo é a umidade do ar, a qual pode afetar a composição química das partículas, modificando parâmetros como o albedo simples e o fator de assimetria (Rosário et al., 2011). Neste sentido, um estudo mais detalhado irá avaliar possí- veis mudanças nas propriedades microfísicas do aerossol, tais como distribuição de tamanho e índice de refração.

\section{Agradecimentos}

Os autores agradecem à NASA, aos grupos de pesquisa e trabalho da rede AERONET e dos sensores MODIS e TRMM, ao CPTEC/INPE e à DSA. Um dos autores agradece o financiamento do $\mathrm{CNPq}$.

\section{Referências}

ALVES, D.S.; DA COSTA, W.; ESCADA, M.I.S.; LOPES, E.S.S; DE SOUZA, R.C.M.; ORTIZ, J.D. Análise da distribuição espacial das taxas de desflorestamento dos municípios da Amazônia Legal no período 1991-1994. $1^{\text {a }}$. ed. São José dos Campos: INPEAMZ- R04/98, 1998. 86 p.

ANDERSON. Liana Oighenstein et al. Sensor MODIS: uma abordagem geral. São José dos Campos: [s.n.], 2003. Disponível em: mtc-m12.sid.inpe.br.

ARTAXO, P.; FERNANDES, E.T.; MARTINS, J.V.; YAMASOE, M.A.; HOBBS, P.V.; MAENHAUT, W.; LONGO, K.M.; CASTANHO, A. Large-scale aerosol source apportionment in Amazonia. Journal of Geophysical Research v. 103, n. D24, p. 31837, 1998.

BECKER, B.K. Amazônia. 5a. ed. São Paulo: Ática, 1997. 112 p.

BECKER, B.K. Estudo elaborado para a Secretaria de Coordenação dos Assuntos da Amazônia Legal. A especificidade do urbano na Amazônia: desafios para políticas públicas consequentes. $1^{\mathrm{a}}$. ed. Rio de Janeiro: Ministério do Meio Ambiente, 1998. p. 60.

BROWN, I.F.; SCHROEDER, W.; SETZER, A.; MALDONADO, M.; PANTOJA, N.; DUARTE, A.; MARENGO, J. Monitoring Fires in Sothwesterns Amazonia Rain Forests. EOS. Transactions, American Geophysical Union v. 87, n. 26, p. 2005-2007, 2006.

CAMPONOGARA, G.; SILVA DIAS, M.A.F.; CARRIÓ, G.G. Relationship between Amazon biomass burning aerosols and rainfall over the la Plata Basin. Atmospheric Chemistry and Physics v. 14, n. 9, p. 4397-4407, 2014.

COELHO, C.A.S.; CAVALCANTI, I.A.F.; COSTA, S.M.S.; FREITAS, S.R.; ITO, E.R.; LUZ, G.; SANTOS, A.F.; NOBRE, C.A.; MARENGO, J.A.; PEZZA, A.B. Climate diagnostics of three major drought events in the Amazon and illustrations of their seasonal precipitation predictions. Meteorological Applications p. 237-255, 2012.

COELHO, C.A.S.; COSTA, S.M.S.; FREITAS, S.R. Diagnóstico do efeito do aerossol na precipitação durante os eventos de seca na Amazônia em 2005 e 2010. In: BORMA, Laura De Simone;; NOBRE, Carlos. (Orgs.). Secas na Amazônia: Causas e Consequências. $1^{\text {a }}$. ed. São José dos Campos: Oficina de Textos, 2013. p. 117-121.

CORREIA, Alexandre; CASTANHO, A.A; MARTINS, V.; LONGO, K. Produto de aerossóis. In: RUDORFF, B. F. T.; SHIMABUKURO, Y.E.; CEBALLOS, J.C. (Orgs.). O sensor MODIS e suas aplicacoes ambientais no Brasil. $1^{\text {a }}$. ed. São Paulo: Oficina de Textos, 2013. 2 v. p. 297-314.

COSTA, Yasmmin Tadeu; RODRIGUES, Silvio Carlos. Efeito do Fogo Sobre a Vegetação e Solo a Partir de Estudo Experimental em Ambiente de Cerrado. Revista do Departamento de Geografia (USP) v. 30, p. 149-165, 2015. 
DOMINGUES, Mariana Soares; BERMANN, Célio. O arco de desflorestamento na Amazônia: da pecuária à soja. Ambiente \& sociedade v. 15 , n. 2, p. 1-22, 2012. Disponível em: http://www.producao.usp.br/handle/BDPI/39759.

EMBRAPA. Tecnologias da Produção de Soja na região central do Brasil. Londrina: [s.n.], 2013. Disponível em: https://ainfo.cnptia.embrapa.br/digi-

tal/bitstream/item/95489/1/SP-16-online.pdf. Acesso em: 10 out. 2016.

ESCADA, MARIA ISABEL SOBRAL; ALVES, DIÓGENES SALAS. Mudanças de Uso e Cobertura do Solo na Amazônia: Impactos Sócio-Ambientais na Ocupação de Regiões de Fronteira Agrícola. São José dos Campos: [s.n.], 2001. Disponível em: http://www.dpi.inpe.br/geopro/modelagem/relatorio_amz_ dinamica_ocupacao.pdf.

FREITAS, S.R.; RODRIGUES, L.F.; LONGO, K.M.; PANETTA,J. Impact of a monotonic advection scheme with low numerical diffusion on transport modeling of emissions from biomass burning. Journal of Advances in Modeling Earth Systems v. 3, n. 1, p. 1-26, 2011

FREITAS, S.R.; LONGO, K.M.; SILVA DIAS, M.A.F.; SILVA DIAS, P.L.; CHATFIELD, R.; PRINS, E.; ARTAXO, P.; GRELL, G.A.; RECUERO, F.S. Monitoring the transport of biomass burning emissions in South America. Environmental Fluid Mechanics v. 5, n. 1-2, p. 135-167, 2005. Disponível em: http://dx.doi.org/10.5094/APR.2011.031.

GODFREY, B.J.; BROWDER, J.O. Disarticulated urbanization in the Brazilian Amazon. The Geographical Review v. 86, p. 441-445, 1996.

GOTO, D.M; LANÇA, M; OBUTI, C.A.; BARBOSA, C.M.G.; SALDIVA, P.H.N.; ZANETTA, D.M.T.; FILHO, G.L.; SANTOS, U.P.; NAKAGAWA, N.K. Effects of biomass burning on nasal mucociliary clearance and mucus properties after sugarcane harvesting. Environmental Research v. 111, n. 5, p. 664-669, 2011.

HOLBEN, B.N.; ECK, I.S.; TANRÉ, D.; BUIS, J.P.; SETZER, A.;VERMOTE, E.; REAGAN, J.A.; KAUFMAN, Y.J.; NAKAJIMA, T.; LAVENU, I.J.; SMIRNOV, A. AERONET - A Federated Instrument Network and Data Archive for Aerosol Characterization. Remote Sensing of Environment v. 66 , n. 1, p. 1-16, 1998. Disponível em: http://www.sciencedirect.com/science/article/pii/S0034425798000315.0034-4257.

HUFFMAN, G.J.; ADLER, R.F.; BOLVIN, D.T.; GU, G.; NELKIN, E.J.; BOWMAN, K.P.; HONG, Y.; STOCKER, E.F.; WOLFF, D.B. The TRMM Multisatellite Precipitation Analysis (TMPA): Quasi-Global, Multiyear, CombinedSensor Precipitation Estimates at Fine Scales. Journal of Hydrometeorology v. 8, n. 1, p. 38-55, 2007.

INSTITUTO NACIONAL DE PESQUISAS ESPACIAIS (INPE). Avaliação da cobertura florestal na Amazônia Legal utilizando sensoriamento remoto orbital. São José dos Campos: Instituto Nacional de Pesquisas Espaciais, 1989.

INSTITUTO NACIONAL DE PESQUISAS ESPACIAIS (INPE). Metodologia para o Cálculo da Taxa Anual de Desmatamento na Amazônia Legal. Disponível em: http://www.obt.inpe.br/prodes/metodologia_TaxaProdes.p df. Acesso em: 25 out. 2014.
INSTITUTO NACIONAL DE PESQUISAS ESPACIAIS (INPE). Monitoramento da floresta Amazônica Brasileira por satélite 1998-1999. São José dos Campos: [s.n.]., 2000

INSTITUTO NACIONAL DE PESQUISAS ESPACIAIS (INPE). Monitoramento da floresta amazônica por satélite 1997-1998. São José dos Campos: [s.n.]., 1999

INTERGOVENAMENTAL PANEL OF CLIMATE CHANGE (IPCC). Climate Change 2013: The Physical Science Basis. Cambridge: [s.n.], 2013. Disponível em: www.climatechange2013.org.

KINTISCH, Eli. Improved Monitoring of Rainforests Helps Pierce Haze of Deforestation. Science, 2007.

KOREN, Ilan; REMER, Lorraine A.; LONGO, Karla. Reversal of trend of biomass burning in the Amazon. Geophysical Research Letters v. 34, n. 20, p. 2-5, 2007.

KUMMEROW, C.; SIMPSON, J.; THIELE, O.; BARNES, W.; CHANG, A.T.C.; STOCKER, E.; ADLER, R.F.; HOU, R.K.; WENTZ, F.; ASHCROFT, P.; KOZU, T.; HONG, Y.; OKAMOTO, K.; IGUCHI, T.; KUROIWA, H.; IM, E.; HADDAD, Z.; HUFFMAN, G.; FERRIER, B.; OLSON, W.S.; ZIPSER, E.; SMITH, E.A.; WILHEIT, T.T.; NORTH, G.; KRISHNAMURTI, T.; NAKAMURA, K. The Status of the Tropical Rainfall Measuring Mission (TRMM) after Two Years in Orbit. Journal of Applied Meteorology v. 39, n. 12, p. 1965-1982, 2000.

LEVY, R.C.; MUNCHACK, L.A.; MATTO, S.; PATADIA, F.; REMER, L.A.; HOLZ, L.E. Towards a long-term global aerosol optical depth record: Applying a consistent aerosol retrieval algorithm to MODIS and VIIRS-observed reflectance. Atmospheric Measurement Techniques v. 8, n. 10, p. 4083-4110, 2015.

LI, J.; CARLSON, B.E.; DUBOVIK, O.; LACIS, A.A. Recent trends in aerosol optical properties derived from AERONET measurements. Atmospheric Chemistry and Physics v. 14, n. 22, p. 12271-12289, 2014.

LONGO, K.M.; FREITAS, S.R.; PIRRE, M.; MARECAL, V.; RODRIGUES, L.F.; PANETTA, J.; ALONSO, M.F.; ROSARIO, N.E.; MOREIRA, D.S.; GÁCITA, M.S.; ARTETA, J.; FONSECA, R.; STOCKER, R.; KATSURAYAMA, D.M.; FAZENDA, A.; BELA, M. The Chemistry CATTBRAMS model (CCATT-BRAMS 4.5): A regional atmospheric model system for integrated air quality and weather forecasting and research. Geoscientific Model Development v. 6, n. 5, p. 1389-1405, 2013.

LYAPUSTIN, A.; SMIRNOV, A.; HOLBEN, B.; CHIN, M.; STREETS, D.G.; LU, Z.; KAHN, R.; SLUTSKER, I.; LAZLO, I.; KONDRAGUNTA, S.; et al. Reduction of aerosol absorption in Beijing since 2007 from MODIS and AERONET. Geophysical Research Letters v. 38, n. 10, p. n/a-n/a, 2011. Disponível em: http://doi.wiley.com/10.1029/2011GL047306.

NOAA. Cold and warm episodes by seasons: EI Niño Southern Oscillation (ENSO) Historical EI Nino/ La Nina episodes (1950-present). Disponível em: http://www.cpc.ncep.noaa.gov/products/analysis_monitoring/ensostuff/ensoyears.shtml. Acesso em: 24 ago. 2016.

PEREIRA, G.; SHIMABUKURO, Y.E.; MORAES, E.C.; FREITAS, S.R.; CARDOZO, F.S.; LONGO, K.M. Monitoring the transport of biomass burning emission in South America. 
Atmospheric Pollution Research n. June 2016, p. 135-167, 2011.

PIRES-NETO, R.C.; SALDIVA, P.H.N.; DOLHNIKOFF, MARISA. Efeitos da Poluição Atmosférica no Epitélio Respiratório das Vias Aéreas Superiores. Revista Brasileira de Medicina v. 2, p. 3-9, 2007.

PROCOPIO, A.S.; ARTAXO, P., KAUFMAN, Y.J.; REMER, L.A.; SCHAFER, J.S.; BOLBEN, B.N.. Multiyear analysis of amazonian biomass burning smoke radiative forcing of climate. Geophysical Research Letters v. 31, p. 1-4, 2004.

PRODES. Levantamento das áreas desflorestadas na Amazônia Legal no período 1991-1994. São José dos Campos: Instituto Nacional de Pesquisas Espaciais. 1996.

REBOITA, M.S.; GAN, M.A.; DA ROCHA, R.P.; AMBRIZZI, T. Regimes de precipitação na América do Sul: uma revisão bibliográfica. Revista Brasileira de Meteorologia v. 25, n. 2, p. 185-204, 2010.

ROSÁRIO, N.E.; YAMASOE, M.A.; BRINDLEY, H.; ECK, T.F.; SCHAFER, J. Downwelling solar irradiance in the biomass burning region of the southern Amazon: Dependence on aerosol intensive optical properties and role of water vapor. Journal of Geophysical Research v. 116, n. D18, p. D18304, 2011. Disponível em: http://doi.wiley.com/10.1029/2011JD015956.

ROSENFELD, Daniel. TRMM observed first direct evidence of smoke from forest fires inhibiting rainfall. Geophysical Research Letters v. 26, n. 20, p. 3105-3108, 1999.

SENA, E. T.; ARTAXO, P.; CORREIA, A. L. Spatial variability of the direct radiative forcing of biomass burning aerosols and the effects of land use change in Amazonia. Atmospheric Chemistry and Physics v. 13, n. 3, p. 1261-1275, 2013.

SETZER, A. Identificação das áreas queimadas nas imagens MODIS. In: RUDORFF, B.F.T.; SHIMABUKURO, Y.E.; CEBALLOS, J.C. (Orgs.). O sensor MODIS e suas aplicações ambientais no Brasil. $1^{\text {a }}$. ed. São Paulo: Oficina de Textos, 2007. p. 297-314.

SILVA, I.C.; PERON, M.C.C.; ARBEX, M.A.; LICHTENFELDS, A.J.F.C.; LOBO, D.J.A.; GIOCONDO, M.P.; MACCHIONE, M.; SAIKI, M.; SALDIVA, P.H.N.; SOARES, C.P. Micronucleus formation induced by biomass burning particles derived from biomass burning induce high micronucleus frequency in Tradescantia pallida assay (TRAD-MN). Journal of the Brazilian Society of Ecotoxicology v. 7, n. 1, p. 1-7, 2012.

SISENANDO, H.A.; MEDEIROS, S.R.B.; ARTAXO, P.; SALDIVA, P.H.N.; HACON, S.S. Micronucleus frequency in children exposed to biomass burning in the Brazilian Legal Amazon region: a control case study. BMC Oral Health v. 12, n. 1, p. 6, 2012. Disponível em: http://www.biomedcentral.com/1472-6831/12.

SISENANDO, H.A.; MEDEIROS, S.R.; SALDIVA, P.H.N.; ARTAXO, P.; HACON, S.S. Genotoxic potential generated by biomass burning in the Brazilian Legal Amazon by Tradescantia micronucleus bioassay: a toxicity assessment study. Environmental Health: A Global Access Science Source v. 10, n. 1, p. 41, 2011. Disponível em: http://www.ehjournal.net/content/10/1/41.

TORRES, O.; CHEN, Z.; JETHVA, H.; AHN, C.; FREITAS, S.R.; BHARTIA, P.K. OMI and MODIS observations of the anomalous 2008-2009 Southern Hemisphere biomass burning seasons. Atmospheric Chemistry and Physics v. 10, p. 3505-3513, 2010.

VENDRASCO, E.P.; SILVA DIAS, P.L.; FREITAS, E.D. A case study of the direct radiative effect of biomass burning aerosols on precipitation in the Eastern Amazon. Atmospheric Research v. 94, n. 3, p. 409-421, 2009. Disponível em: http://dx.doi.org/10.1016/j.atmosres.2009.06.016.

YAMASOE, M.A.; RANDOW, C.V.; MANZI, A.O.; SCHAFER, J.S.; ECK, T.F.; HOLBEN, B.N. Effect of smoke and clouds on the transmissivity of photosynthetically active radiation inside the canopy. Atmospheric Chemistry and Physics v. 6, p. 1645-1656, 2006. Disponível em: https://hal.archives-ouvertes.fr/hal-00295923.

ZHANG, J.; REID, J.S. A decadal regional and global trend analysis of the aerosol optical depth using a data-assimilation grade over-water MODIS and Level 2 MISR aerosol products. Atmospheric Chemistry and Physics v. 10, n. 22, p. 10949-10963, 2010.

This is an Open Access article distributed under the terms of the Creative Commons Attribution Non-Commercial License which permits unrestricted non-commercial use, distribution, and reproduction in any medium provided the original work is properly cited. 